\title{
Work Related Musculoskeletal Symptoms among Metro Rail Workers in Nagpur City
}

\author{
Harshadeep Kothare ${ }^{1}$, Kalyani Shakkarwar ${ }^{2}$ \\ ${ }^{1}$ Assistant Professor, VSPMs College of Physiotherapy, Digdoh Hills, Hingna Road, Nagpur, Maharashtra, India \\ ${ }^{2}$ VSPM's College of Physiotherapy, Digdoh Hills, Hingna Road, Nagpur, Maharashtra, India
}

Corresponding Author: Harshadeep Kothare

\begin{abstract}
Background: The Nagpur Metro rail project workers are involved in heavy activities like pulling, pushing, lifting heavy loads and other heavy tasks. This makes them susceptible to Musculoskeletal disorders (MSDs)/symptoms.

Aim and Objectives: To find out the presence of work related musculoskeletal symptoms in Metro workers in Nagpur city using Nordic musculoskeletal questionnaire.

Methods: 120 workers falling, in the age range of 20 to 45 years and involved in labor work were included using the convenient sampling method. Samples were excluded if they have recent fractures or surgery, any neurological conditions or who were not willing to participate. Nordic Musculoskeletal questionnaire was explained to each and every worker in their vernacular language.

Results and Conclusion: The result showed that there is multiple region involvement among the subjects. The most affected region was found to be upper back followed by low back, knee, shoulder, neck.
\end{abstract}

Keywords: Metro Rail Workers, Work related Musculoskeletal Disorders.

\section{INTRODUCTION}

The Nagpur Metro rail project was started on February 2015. The types of workers involved in project include Engineers, project engineers, station managers, supervisors and workers (labourers) etc. Among these, the workers/ labourers are involved heavy activities like pulling, pushing, lifting heavy loads and other heavy tasks ${ }^{1}$.
These types of works are likely to cause musculoskeletal symptoms in those workers.

Workers who are performing heavy physical work have a significantly higher prevalence of Musculoskeletal disorders (MSDs)/symptoms in different regions of the body ${ }^{1}$.

In many types of occupational groups, MSDs are major causes of work related disability ${ }^{1}$

Common musculoskeletal disorders include Carpal tunnel syndrome, Muscle/ Tendon strain, Ligament sprain, Tension neck syndrome, Thoracic outlet syndrome, Rotator cuff tendonitis, Lateral Epicondylitis, Radial tunnel syndrome, Trigger finger/Thumb, Mechanical back syndrome, Degenerative disc disease, Rupture disc ${ }^{2}$.

Workplace and the nature of work are factors that have significant relationship with back pain due to the perception that the load carried at work is too heavy ${ }^{3}$ Anuar I et.al. observed that prevalence of back pain remains high among people carrying works such as drilling, lifting, pushing, pulling which involve body twisting, especially bending ${ }^{4}$

Work related activities and working in awkward posture are the most frequently reported work- related activities that caused musculoskeletal symptoms among construction workers ${ }^{5}$.

Working in the same position for long periods was the job factor identified as most problematic, where most respondents 
rated as a major problem contributing musculoskeletal sypmtoms ${ }^{6}$. In addition, certain postures can be assumed safe if they occur for a short period, provided the workers do not repeat that posture \& classify such postures as unsafe when a time threshold has been met ${ }^{7}$.

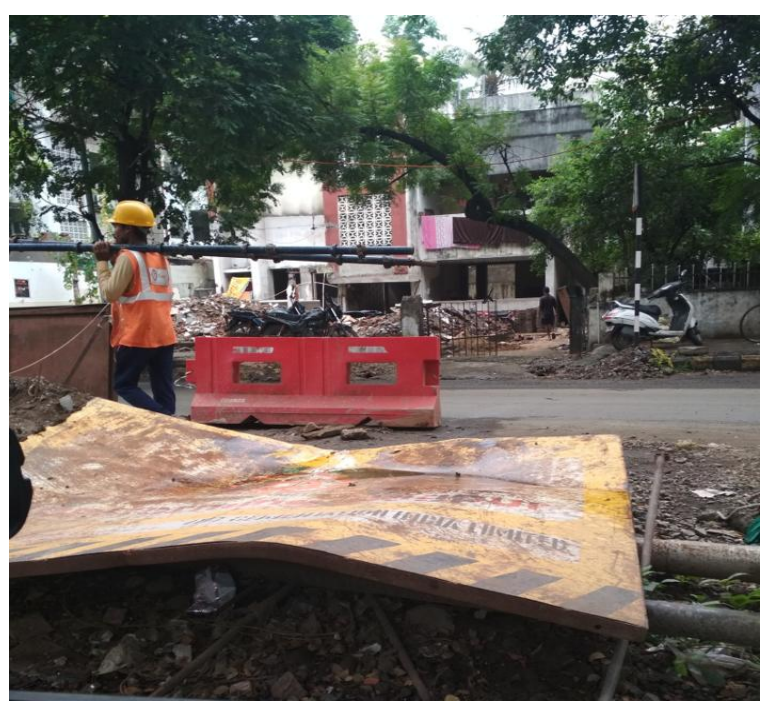

Aim and Objective

To determine the work related musculoskeletal symptoms at different body sites in metro workers using Nordic musculoskeletal questionnaire.

\section{METHODOLOGY}

Study setting: various metro stations in Nagpur city.

\section{Target study design: Observational study}

Population: Metro Rail workers

Sample size: 120

Type of sampling: Convenient sampling

\section{Inclusion criteria}

- Metro Workers involved in labor activities.

- Working since 6 months or more.

- Age-20 to $45 \mathrm{yrs}$.

\section{Exclusion criteria}

- Recent fracture, surgery.

- Any neurological condition.

- Subjects not willing to participate

\section{General Procedure}

The requisite permission for carrying out the study was obtained before the commencement of the study from the concerned authorities.

Preparation of the study protocol and obtained the ethics clearance for the research.

The study protocol required the use of Nordic musculoskeletal questionnaire.

The study protocol was explained in detail to the workers who would be interested to be the part of the study.

Written consent was obtained from the participants in accordance to the guidelines of ICMR after they were convinced with the subject information provided to them. The workers who are interested to be the part of the study had submitted the written informed consent duly filled.

Care was taken to provide adequate time for completing the questionnaire. The completed questionnaire was analyzed for the responses and the responses obtained from the questionnaire were entered in Microsoft excel office professional plus sheet 2007.

The raw data was later analyzed with appropriate statistical test and identifying the significance level was done.

\section{Data analysis}

The collected data was expressed in MS Excel Sheet.

The body site wise distribution of musculoskeletal symptoms was expressed in the form of tables and graphical representation.

\section{RESULTS}

Table 1: Age Distribution of subjects

\begin{tabular}{|c|c|c|}
\hline Age & Frequency & Percent \\
\hline $20-24$ & 26 & 21.7 \\
\hline $25-29$ & 24 & 20.0 \\
\hline $30-34$ & 25 & 20.8 \\
\hline $35-39$ & 25 & 20.8 \\
\hline $40-44$ & 20 & 16.7 \\
\hline Total & $\mathbf{1 2 0}$ & $\mathbf{1 0 0 . 0}$ \\
\hline
\end{tabular}




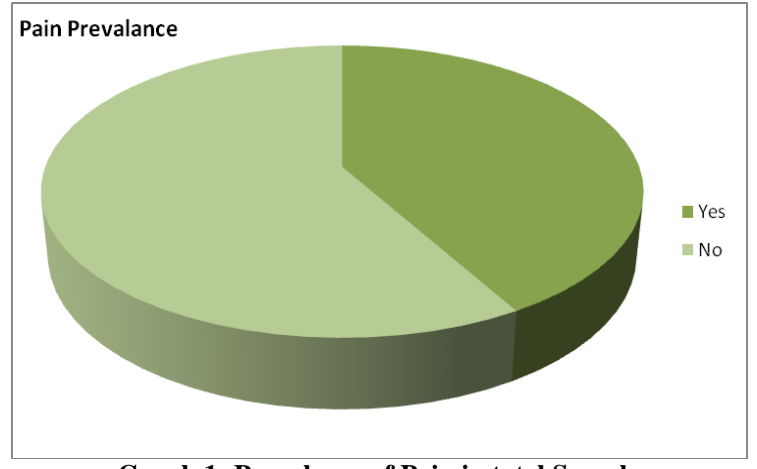

Graph 1: Prevalence of Pain in total Sample
Table 2 and Graph 2 shows that during the last 12 months, most subjects had trouble (such as ache, pain, discomfort, numbness) in Upper Back (45\%) followed by Lower Back (20\%), Knee (11.7\%), Shoulder (6.7\%), Neck (5\%), Elbows and Hips $(3.3 \%$ each) and least in Ankle and wrist $(2.5 \%)$

\begin{tabular}{|c|c|}
\hline KNEE & $\mathbf{1 1 . 7}$ \\
\hline Lower back & 20 \\
\hline Shoulder & 6.7 \\
\hline Neck & 5 \\
\hline Ankle & 2.5 \\
\hline Hip & 3.3 \\
\hline Elbow & 3.3 \\
\hline Wrist & 2.5 \\
\hline Upper back & 45 \\
\hline
\end{tabular}

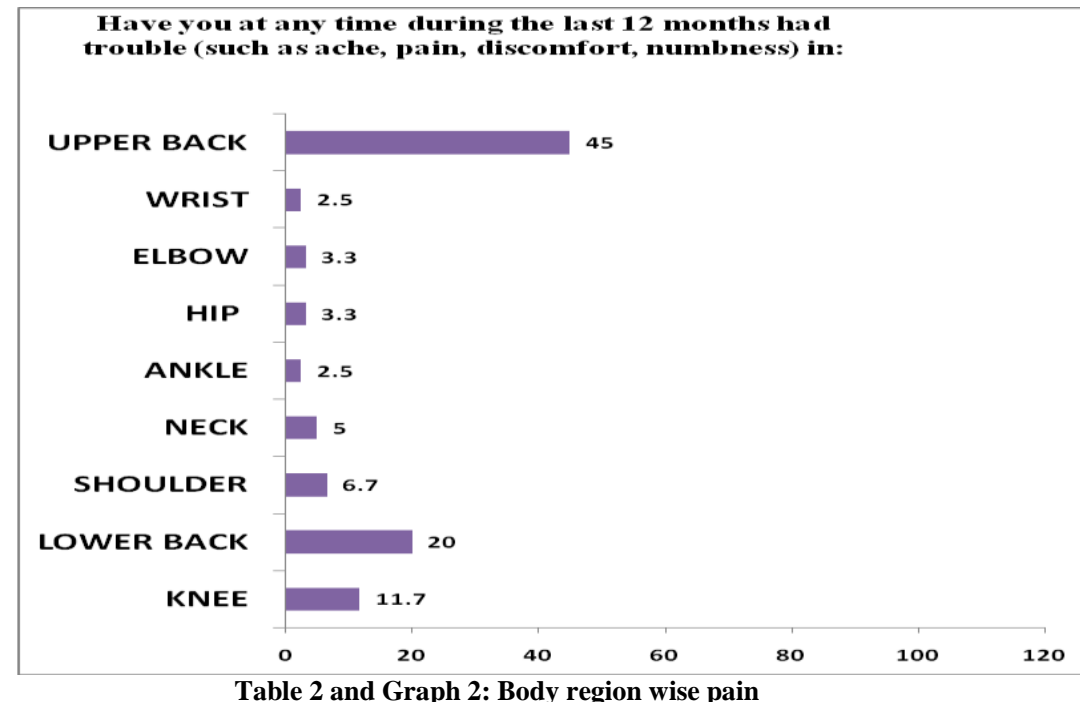

\begin{tabular}{|c|c|}
\hline KNEE & $\mathbf{4 0}$ \\
\hline Lower back & 66.7 \\
\hline Shoulder & 30 \\
\hline Neck & 10 \\
\hline Ankle & 10 \\
\hline Hip & 30 \\
\hline Elbow & 20 \\
\hline Wrist & 20 \\
\hline Upper back & 58 \\
\hline
\end{tabular}

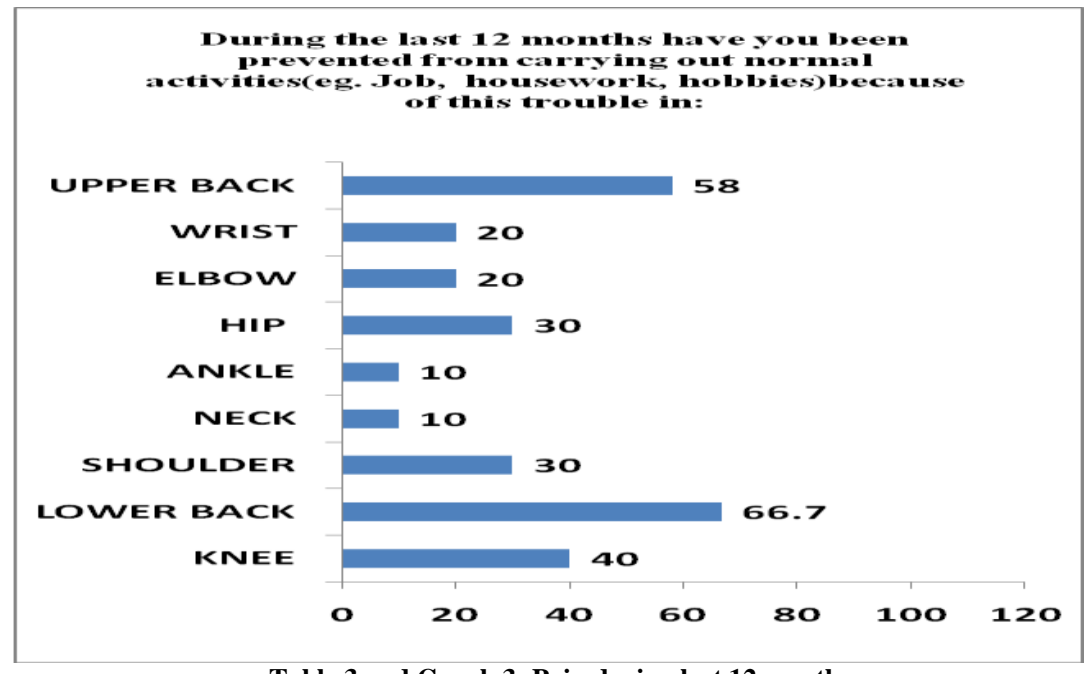

Table 3 and Graph 3: Pain during last 12 months

Table 3 and Graph 3 shows that during the last 12 months most subjects have been prevented from carrying out normal activities (e.g. Job, housework, hobbies) because of this trouble in Lower back $(66.7 \%)$ followed by Upper back $(58 \%)$, Knee (40\%), Hips and Shoulders (30\% each), Wrist and Elbows (20\% each) and least in Ankle and Neck (10\% each)
Table 4 and Graph 4 shows that during the last months the subjects have seen a physician for trouble in Upper Back (100\%), Lower Back (83.3\%), Knees (15.4\%), Hips and Shoulders (20\% each) and Wrist, Elbow, Ankle and Neck (10\% each). 


\begin{tabular}{|c|c|}
\hline KNEE & $\mathbf{1 5 . 4}$ \\
\hline Lower back & 83.3 \\
\hline Shoulder & 20 \\
\hline Neck & 10 \\
\hline Ankle & 10 \\
\hline Hip & 20 \\
\hline Elbow & 10 \\
\hline Wrist & 10 \\
\hline Upper back & 100 \\
\hline
\end{tabular}

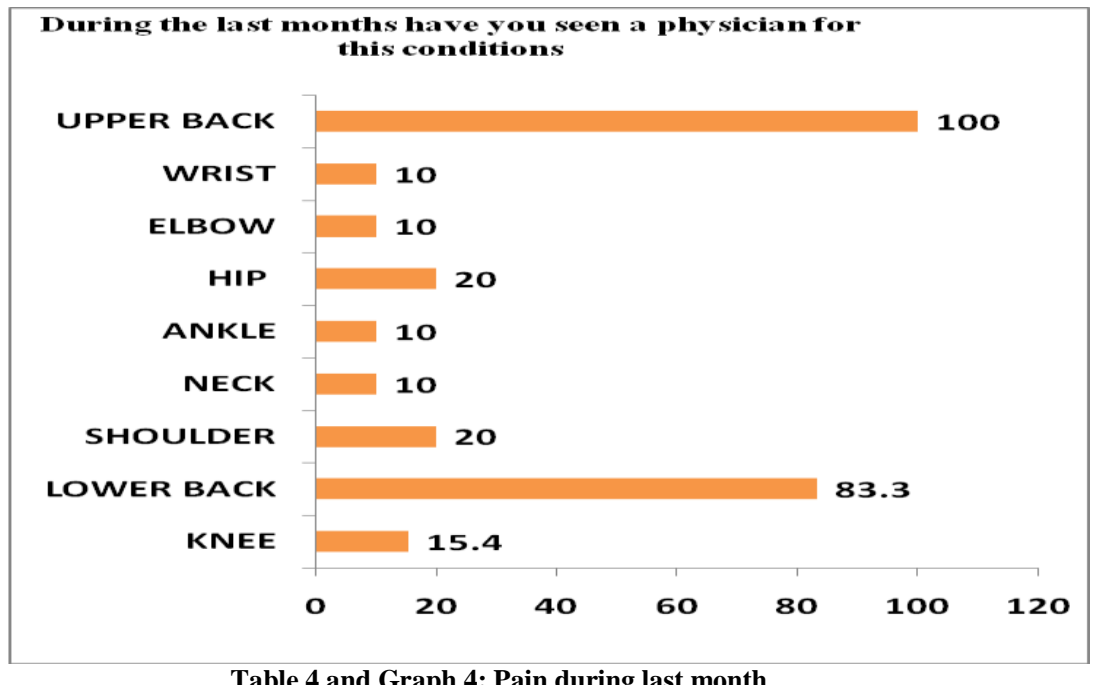

\begin{tabular}{|c|c|}
\hline KNEE & $\mathbf{2 5 . 5}$ \\
\hline Lower back & 75 \\
\hline Shoulder & 38.5 \\
\hline Neck & 11.5 \\
\hline Ankle & 20 \\
\hline Hip & 40 \\
\hline Elbow & 10 \\
\hline Wrist & 15.5 \\
\hline Upper back & 100 \\
\hline
\end{tabular}

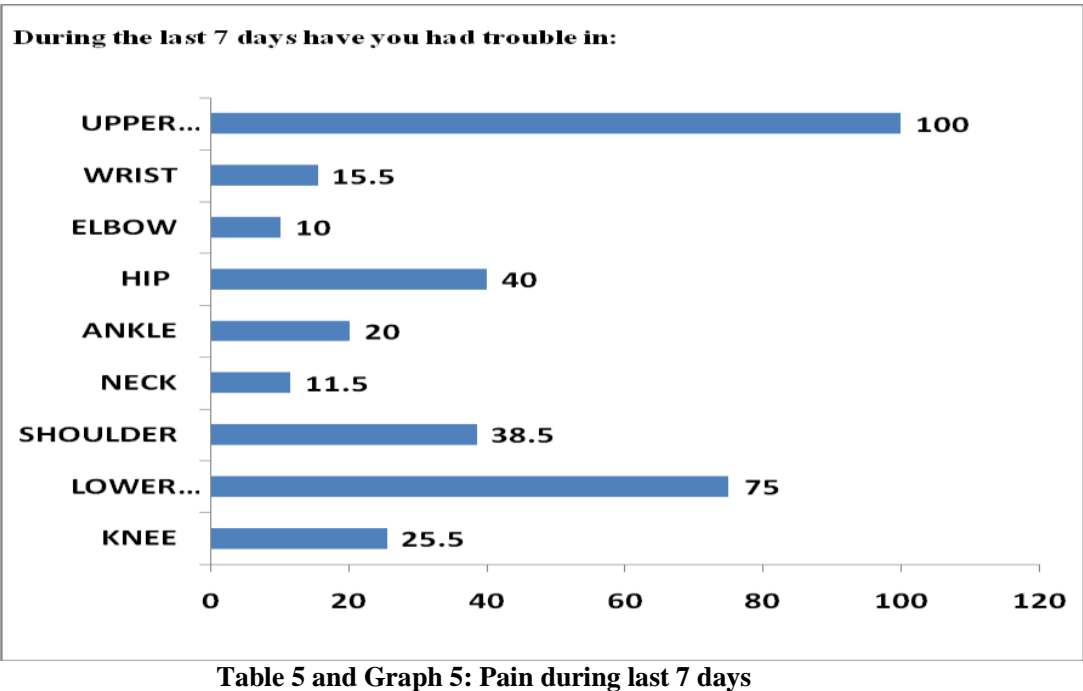

Table 5 and Graph 5 shows that during the last 7 days the subjects had trouble mostly in Upper Back (100\%) followed by Lower Back (75\%), followed by Hip (40\%), Shoulder (38.5\%), Knee (25.5\%), Ankle (20\%), Wrist (15.5\%), Neck (11.5\%) and Elbow (10\%).

\section{DISCUSSION}

The present study was carried out to identify the musculoskeletal pain in Nagpur Metro Rail construction workers.

The results in the present study showed that there is multiple region involvement among the subjects. The most affected region was found to be upper back followed by low back, knee, shoulder, neck. ${ }^{8}$
Off all the respondents who reported WRMSDs, a variable number reported have noted a physician for the treatment was $45 \%$ with upper back pain problem, with $15 \%$ of those with shoulder ,20\% of lower back, $10 \%$ with wrist, $5 \%$ of hip,5\% of ankle/feet problems. ${ }^{9}$

Majority of the participant in the present study had upper back pain during the last 12 months(45\%). This is in consistence with study of Kalenial et al. who started that majority of subjects have upper back pain.

Whereas the study conducted in (2002) Yang et al. found that the shoulder is the most frequently reported body region with musculoskeletal symptom. However most of the studies revealed upper back symptoms as the most prevalent 
musculoskeletal complaints among workers. ${ }^{11}$

Lifting and transferring heavy loads were the job activities most common in metro workers. So, this might be factor for the most of the respondent have upper back pain. $^{12}$

Metro workers work almost all day mostly in standing. Workers who have to work standing for prolonged period of time have higher risk of back symptoms. ${ }^{13}$

There is a greater amount of compressive force on the spine and repetitive motion is responsible for the back pain. Due to prolonged stress, there is strain on muscles and ligaments which decrease their ability to support spine. ${ }^{14}$

Few studies, shown that psychosocial factors and stress are associated with the musculoskeletal symptoms. ${ }^{15}$

\section{CONCLUSION}

From this study, it can be concluded that the metro station worker work for long duration and their work load may result in the development of musculoskeletal disorders. Most common region involved upper back followed lower back, knee and shoulder.

\section{Acknowledgement: None}

\section{Conflict of Interest: None}

\section{Source of Funding: None}

\section{Ethical Approval: Approved}

\section{REFERENCES}

1. Chang FL, Sun YM, Chuang KH, Hsu DJ (2009).Work fatigue and physiological symptoms In different occupations of highelevation construction workers. Appl Ergon, 40: $591-596$

2. Ergo-plus.com/Musculoskeletal disordersmdd/by Matt Middles worth.

3. Rahmah MA, Rozy IHJ, Jamiah M, Shamsu AS(2008). Prevalence of Back Pain Among Nurses Working in Government Health
Clinics and Hospital in Port Dickson, Malaysia. J Comm Health, 14(2): 1-8

4. Anuar I, Nurulakhmar AS, Mazrura S, Azhar AH(2010). Study On Backpain And The Contributing Factors Among Workers In Automotive Industry. J Comm Health, 6(2): 1-7

5. Yang YS, Goldsheyder D, Kau LJ (2002). Survey of Musculoskeletal Symptoms among Building Construction Workers in Southern Taiwan. JOccup Therapy Assoc, R.O.C., 20:12-20.

6. Medino LA, John CR, Dan A, Thomas MC (2003). Symptoms of Musculoskeletal Disorders among Apprentice Construction Workers. Appl Occup and Environ Hygiene, 18(1): 57-64.

7. Ray SJ, Teizer J (2012). Real-time construction worker posture analysis for ergonomics training. Adv Eng Inform, 26: 439- 455

8. Kant I, Notermans JHV, Borm PJA. Observations of working posture in garages using Ovako Working Posture Analysing System (OWAS) and consequent workload reduction recommendations. Ergonomics 1990, 33:209-220.

9. Silverstein B, Vikari-Jutura E, Kalat J. Use of preventive index to identify industries at high risk for work-related musculoskeletal disorders of the neck, back and upper extermity in Washington state,19901998.Am J Ind Med 2002;41-169.

10. Buckle PW, Devereux JJ. The nature of work related neck upper limb musculoskeletal disorders. Appl Ergon 2002z, 33;207-217.

11. Gangopadhyay S, Das B, Das T, Ghoshal G,Ghosh T (2010). An Ergonomics Study on Posture-Related Discomfort and Occupational Related Disorders Among Stonecutters of West Bengal, India. Int $J$ Occup Safety and Ergon, 16: 69 -79.

12. Moradi-Lakeh M, Forouzanfar MH, Vollset SE, Bcheraoui C, Daoud F, Afshin A et al. Burden of musculoskeletal disorders in the Eastern Mediterranean Region, Ann Rheum Dis $2017 ; 1365-1373$.

13. Abledu JK, Offei EB. Musculoskeletal disorders among first-year Ghanaian students in a nursing college. Afr Health Sci 2015; 15:444-449.

14. Couture L. Health problems related to standing. Commission de la Sante et de la 
Sequrite du Quibec (CSST), CREE (report \#86082901), 1986.

15. Salian Shivani Chowdhury, Jinal Boricha, and Sujata Yardi. Identification of awkward postures that cause discomfort to liquid petroleum gas workers in Mumbai, India. Indian J Occup Environ Med. 2012 JanApr;16(1):3-8.
How to cite this article: Kothare H, Shakkarwar $\mathrm{K}$. Work related musculoskeletal symptoms among Metro Rail workers In Nagpur City. International Journal of Science \& Healthcare Research. 2021; 6(3): 7-12. DOI: https:// doi.org/10.52403/ijshr.20210702 